\title{
Changes in EMG activity during clenching in chronic pain patients with unilateral temporomandibular disorders
}

\author{
U. Santana-Moraa, J. Cudeiro, M.J. Mora-Bermúdez, B. Rilo-Pousa, J.C. Ferreira-Pinho, J.L. \\ Otero-Cepeda, U. Santana-Penín,
}

\begin{abstract}
The study assessed the differences in electromyographic (EMG) activity recorded during clenching in women with chronic unilateral temporomandibular disorders (TMDs) as compared to control subjects. Seventy-five full dentate, normo-occlusion, right-handed, similarly aged female subjects were recruited. Twenty five subjects presented with right side TMD, 25 presented with left side TMD and 25 pain-free control subjects participated. Using integrated surface EMG over a $1 \mathrm{~s}$ contraction, the anterior temporalis and masseter muscles were evaluated bilaterally while subjects performed maximum voluntary clenching. Lower EMG activation was observed in patients with TMD as compared to control subjects (temporalis: $195.74 \pm 18.57$ vs. $275.74 \pm 22.11, \quad P=0.011$; masseters: $151.09 \pm 17.37$ vs. $283.29 \pm 31.87, \quad P<0.001)$. An asymmetry index (SAI) was calculated to determine ratios of right to left sided activation. Patients with right-sided TMD demonstrated preferential use of their left-sided muscles (SAI $-5.35 \pm 4.02)$ whereas patients with left-sided TMD demonstrated preferential use of their rightsided muscles (SAI $6.95 \pm 2.82)$, $(P=0.016)$. This unilateral reduction in temporalis and masseter activity could be considered as a specific protective functional adaptation of the neuromuscular system due to nociceptive input. The asymmetry index (SAI) may be a useful measure in discriminating patients with right vs. left-sided TMD.
\end{abstract}

Keywords: Unilateral TMD; EMG; Jaw muscles; Clenching; Pain

\section{Introduction}

Pains that originate from the musculoskeletal structures of the masticatory system are included in a category of pain complaints collectively known as temporomandibular disorders [(TMD); (Okeson, 1996 and Liljeström et al., 2005)]. Pain in the temporomandibular region appears to be relatively common, occurring in approximately $10 \%$ of the population over age 18 ; it is primarily a condition of young and middle-aged adults, rather than of children or the elderly, and is approximately twice as common in women as in men (LeResche, 1997). The exact causes of most TMDs, with the exception of the traumatic aetiologies, remain either largely unknown or are speculative (Greene, 2006 and Tanaka et al., 2008).

Subjects with TMD-pain alter the recruitment of their jaw muscles (Nielsen et al., 1990). Free nerve endings act as nociceptors activated by noxious stimulation such as temporomandibular joint (TMJ) overloads and/or masticatory muscles ischemia, if it is prolonged and associate with muscle contractions (Milam et al., 1998, Sessle, 2000a and Tanaka et al., 2008). A decrease of motor unit firing rate has been correlated to the intensity of muscle pain, but the central mechanisms involved remain unclear (Farina et al., 2004). Maximum EMG activity is greater in pain-free subjects than in patients with TMD pain (Helkimo et al., 1975 and Fogle and Glaros, 1995). Though substantially lower bite-force has been observed in TMD patients than in controls, such force is similar on the disordered and non-disordered sides (Molin, 1972). The maximum bite-force of masticatory muscles can be generated during clenching (Koolstra et al., 1988a), and consequently the greatest EMG values may be registered (Van Eijden et al., 1993, Erhardson et al., 1993 and Wood, 1987). The bite-force increases in relation to muscle activity (Hidaka et al., 1999). Muscle forces act on the structures of the masticatory apparatus and may generate excessive loading on the tooth row and/or TMJs (May 
and Garabadian, 2000). Maximal bite-force magnitude is mainly dependent on factors associated with the masseter muscles (Koolstra et al., 1988a, Koolstra et al., 1988b and Van Spronsen et al., 1996), and to a lesser extent, other craniofacial factors (Raadsheer et al., 1999). In order to avoid overloading, the clenching bite-force adjusts to a position where it is well-balanced (Hidaka et al., 1999), and muscle forces are directed to minimize joint loads and muscle efforts (Nickel et al., 2003).

Though mathematical and biomechanical models have explained the effect of the force of masticatory muscles (Smith et al., 1986, Koolstra et al., 1988a and Ferrario and Sforza, 1994), in vivo studies of EMG activity are required to substantiate the findings of these models given that the observations of in vitro models may not be extrapolated to complex clinical contexts.

To our knowledge, the magnitude of EMG activity of the jaw muscles in patients with chronic, unilateral TMD has not been documented, and the diagnostic value of EMG tests is yet to be established (Gonzalez et al., 2008 and Suvinen and Kemppainen, 2007). This crosssectional study of clench-EMG activity of jaw muscles assessed three null hypotheses: (1) there are no differences between symptom-free and unilateral TMD subjects, (2) there are no differences between patients suffering from right side TMD in contrast to those suffering from left side TMD, and (3) there are no differences between the pain and non-pain sides of unilateral TMD patients.

\section{Material and methods}

\subsection{Subjects}

Since jaw elevator muscle activity can be influenced by orofacial pain (Helkimo et al., 1975, Fogle and Glaros, 1995 and Svensson et al., 2004), gender (Sessle, 2000b, Johansson et al., 2003 and Sarlani and Greenspan, 2005), age (Fogle and Glaros, 1995), occlusion (Carlsson and Ingervall, 1988, Møller and Bakke, 1988, Racich, 2005 and del Palomar et al., 2008), and hemispheric dominance (Pirttiniemi, 1998), this study evaluated 75 full-dentate (except for third molars), normal-occlusion, right-handed (7-10 points in Edinburgh inventory; Oldfiel, 1971), similarly aged (18-22 years), female subjects (inclusion criteria). All subjects (except one control) presented premature occlusal contacts.

Exclusion criteria were: periodontal pathology, pain, bleeding and/or $3 \mathrm{~mm}$ pockets probing; dental decay, caries or damaged dental tissues; fixed restorations that affected occlusal surfaces; clinically visible malocclusions; orthodontic therapy in the lasts two years except passive retention; bruxism, diagnosed by the presence of parafunctional facets and/or anamnesis of parafunctional tooth clenching and/or grinding; neuropathic conditions evaluated, when indicated, by neurologist; systemic and/or localized maxillofacial disease; Botox therapy; psychological disorders (when indicated, after examination by a psychologist/psychiatrist); and pregnancy.

Subjects in the patient group were randomly selected from 273 patients who attended the TMD clinic of the Faculty of Medicine and Dentistry of University of Santiago de Compostela, Spain, from February 1997 to July 2005. Using a VAS, patients were asked to mark one point which represented the intensity of self-reported pain on a non-graded straight line of $10 \mathrm{~cm}$, from non pain to the worst pain imaginable (Carlsson, 1983). Eligible subjects were female patient who presented a major complaint of painful unilateral TMD, and diagnosed as chronic-pain Axis I (Dworkin and LeResche, 1992). All patients showed TMD chronic-pain for over six months (Merskey and Bogduk, 1994). The largest reported pain period was five years. No differences were found between patient's subgroups regarding both the length of the pain duration or the kind of the previous therapy.

According to the aforementioned criteria, 50 subjects (25 patients had right TMD and 25 left TMD) were included for study (20.46 \pm 1.34 years). Magnetic Resonance Imaging (MRI) was indicated in painful unilateral TMD patients when sign/s, such as altered jaw movements and/or TMJ sounds were present, suggesting internal derangements (Brooks et al., 1997). MRI studies have permitted the classification of patients into two major groups (IHS classification 11.7, 11.8, 1998): pain of articular origin-artralgia and pain of muscular origin-myalgia (Okeson, 1996). Fifteen patients were studied with MRI.

The control group (pain-free subjects) was composed of 25 odontology students $(20.40 \pm 1.32$ years) with no clinical pathology or history of TMD symptoms.

An institutional review board approved the project, and each subject gave informed consent. 


\subsection{EMG activity analysis}

Standards for Reporting EMG Data from International Society of Electrophysiology and Kinesiology (Merletti, 1999) were followed as the protocol to perform EMG studies.

Electrodes were positioned on the skin parallel to the direction of the muscle fibers (Fig. 1). Motor point of the muscles was determined by visual observation and palpation. When bipolar electrodes straddle the motor point, the negative ones were displaced towards the most bulky part of the long head of each muscle. The ground surface electrode was taped below the subject's left earlobe.

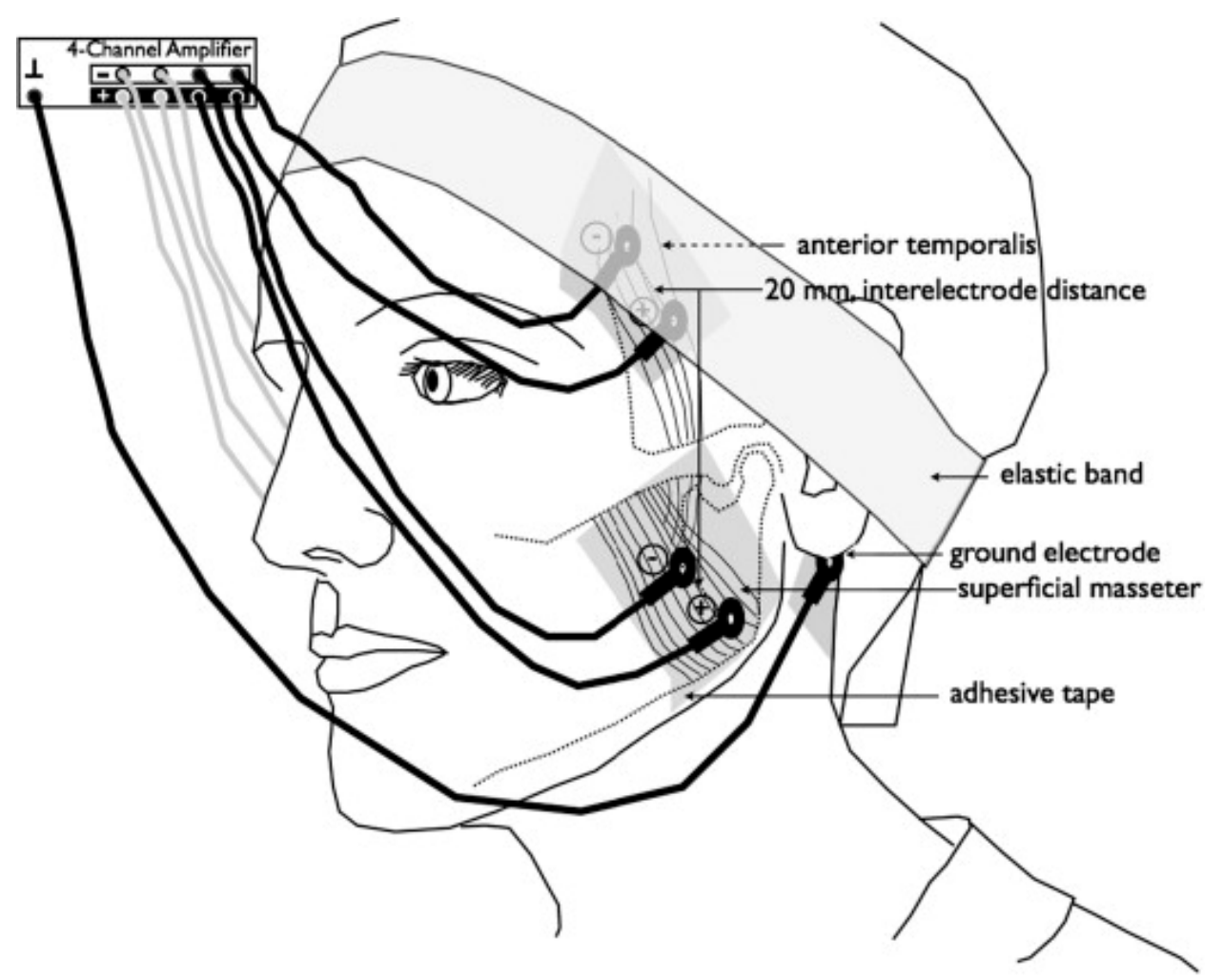

Fig. 1. Electrode location

After the skin was degreased (Nuprep ${ }^{\mathrm{TM}}$, D.O. Weaver and Co., Au, USA) and rubbed with grinding paper to reduce electrode-skin impedance, surface EMG signals were obtained with 10$\mathrm{mm}$ bipolar gold surface electrodes (Nicolet Biomedical, Madison, WI, USA) with 2-cm electrode distance. To minimize electrode movements during the study, electrodes were located in place while the patient performed submaximal muscular contraction. Since the presence of hair limited the adhesion of the pads and created noises an elastic band was placed over the temporalis electrodes. Careful attention was paid to the correct positioning of electrodes during the examination. The ground surface electrode was taped below the subject's left earlobe. Conductive paste (Ten20 ${ }^{\mathrm{TM}}$, D.O Weaver and Co., Au, USA) was applied filling the electrodes and then were attached and secured by adhesive pads (Omnifix, Paul Hartmann Corp., ILL, USA). Electrodes were connected to the Nicolet Viking Select electrodiagnostic system (Nicolet Biomedical, Madison, WI, USA) at $200 \mathrm{~ms}$ time base by $200 \mu \mathrm{V}$ to $1 \mathrm{mV}$ of amplitude. The patient was seated on a comfortable chair without head support with the trunk in an erect posture and the head in the natural position. The electrodes were connected to the 4-channel amplifier, placed above the subject, approximately $50 \mathrm{~cm}$ a part, avoiding the effect of gravity and inertia on electrodes and cables. Calibrated surface impedances were lower than $5 \mathrm{k} \Omega$. The differential raw EMG signal was filtered and amplified (band pass $20-1 \mathrm{kHz}$, sampling frequency $2 \mathrm{kHz}$ ). Rectification and smoothing with a low-pass filter was performed with hardware prior to sampling and storing data; sampling rate to introduce the EMG envelope into the computer was $250 \mathrm{~Hz}$. 
Real time screen displayed EMG-records were used as a biofeedback tool to standardize a more symmetrical and minimal active rest position, and most symmetrical and high clenching level in maximal intercuspal position (Cram, 2004).

The EMG recordings evaluated the muscle output during sustained maximum voluntary clenching for over $1 \mathrm{~s}$ and less than $4 \mathrm{~s}$, repeated three times per session with a 5-min rest interval, and were undertaken in a quiet environment at $21^{\circ} \mathrm{C}$ with only the explored subject seated in a raised comfortable position on an examination chair. Surrounding noises were controlled. One experimentally blind operator, moving as little as possible during the experiment, performed EMG recordings. Subjects were instructed to maintain a sustained maximum voluntary clenching avoiding any facial or orbicular expression or jaw or head movement. All signals were viewed on a display screen prior to collection to ensure that there were no visible artifacts.

Computer analysis of the EMG data displayed bioelectric activity in area units, $\mu \mathrm{V} \mathrm{X} \mathrm{ms,} \mathrm{and}$ permitted storage and quantification of the area between basal and peak lines during ascertain time (Fig 2). Magnitude of bioelectric activity for one second was calculated after manual positioning of the cursors in the central part of maximum displayed EMG activity. A mean of the three records by subject was the value used.
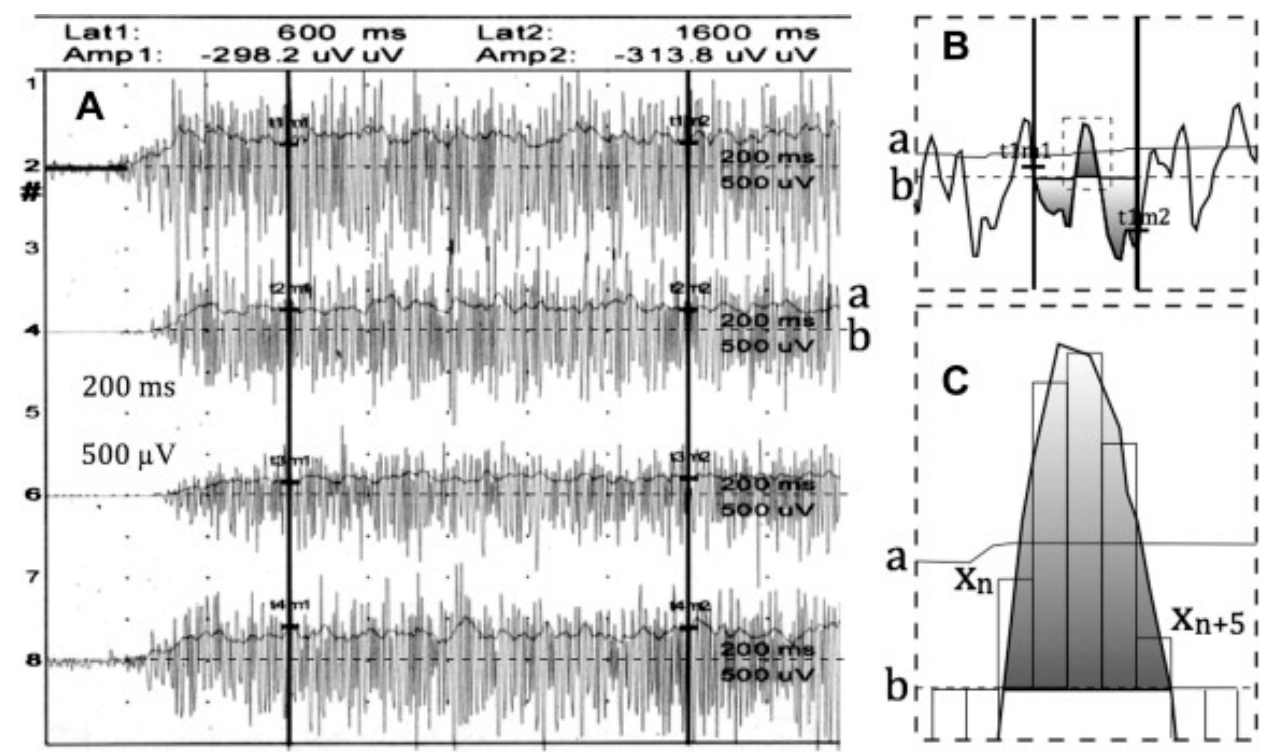

Fig. 2. Description of the EMG-activity area undergoing computer analysis. A, EMG-picture with bars limiting one second of activity; B, C, detail of the area automatically calculated by the Nicolet system. a, mean values line; b, base-line.

The system performs the following algorithm:

$$
\text { Area }=\sum_{x=t_{1} m_{1}}^{t_{1} m_{2}-1}\left(\mid A m p(x)^{*} \text { time Pr Sample } \mid\right)
$$

Calculation of the mean amplitude value requires the division of the EMG-magnitude (integral of the rectified signals for one second, in $\mu \mathrm{V} \mathrm{X} \mathrm{ms)} \mathrm{by} 2.000$ (sampling rate, along one second).

To assess EMG-reproducibility, three records per session were taken in two sessions (separated by one to four weeks) carried out in 10 randomly selected control subjects. 


\subsection{Index calculation}

The asymmetry index between sides (SAI) was calculated to determine ratios of right to left sided activation, obtained by the equation (Naeije et al., 1989):

$\mathrm{SAI}=\frac{\text { right } \text { masseter }=\text { right temporalis }- \text { left masseter }- \text { left temporalis }}{\text { right } \text { masseter }=\text { right temporalis }- \text { left masseter }+ \text { left temporalis }} \times 100$

\subsection{Statistical analysis}

Data are reported as mean \pm standard error (SE) of the mean. EMG activity data acquisition, evaluation, and statistical analysis were performed under blind conditions. A Pearson correlation coefficient was used to detect the possible association of EMG activity with the clinical VAS scores. Because no quantitative EMG-variables showed violation for normality and homoscedasticity, repeated-measures analysis of variance (ANOVA) were performed to assess dependent variables (EMG values and indices of the four muscle sites) of the unilateral TMD, left TMD and right TMD subgroups and the control group (Kleinbaum et al., 1988). When ANOVA indicated a significant difference, Scheffé test was performed as post-hoc test. The level for significance was set at $P$ value $=0.05$. Intraclass coefficient correlation (ICC) was calculated to estimate the intra- and inter-sessional reproducibility ( Castroflorio et al., 2006).

\section{Results}

\subsection{VAS scores vs. EMG-activity}

A total of 50 patients had a major complaint of spontaneous pain: the VAS mean score was $4.76 \pm 1.70$ (range, 3-10). VAS scores were not correlated with the EMG activity of the jaw muscles: Pearson's correlation revealed no significant $(0.104 \leqslant P \leqslant 0.476)$ negative values (from -0.103 for the right masseter to -0.233 for the left temporalis).

Thirteen patients showed pain of articular origin (seven patients with right TMD and six patients with left TMD), the other ones were classified as muscular disorders.

\subsection{EMG activity}

\subsubsection{Reproducibility}

In the first session, the ICC mean for the four muscles was 0.931 ; in the second session was 0.906; and globally, ICC $=0.886$.

\subsubsection{Control subjects and patients (Table 1 and Fig. 3)}

Mean EMG-clench activity for pain-free subjects was $279.52 \pm 24.48 \mathrm{mV} \times \mathrm{ms}$; no differences were observed between the four muscles in the control group $(P=0.387)$. Mean EMG-clench activity for patients was $173.42 \pm 17.17 \mathrm{mV} \times \mathrm{ms}$, with a significant difference between the four muscles ( $P=0.001$, ANOVA-test). No differences in EMG activity were found between patients with pain of articular origin and pain of muscular origin in the four muscle sites.

EMG values for patients was significantly lower $(P=0.001)$ than for the control group. This affects specifically to temporalis (patients $195.74 \pm 18.57$ vs. control $275.74 \pm 22.11, P=0.011$ ), and masseter muscles (patients $151.09 \pm 17.37$ vs. control $283.29 \pm 31.87, P<0.001$ ). 
Table 1. Mean \pm SE of EMG-activity of four sites, in $\mathrm{mV} \times$ ms units. $P_{1}$, right TMD vs. left TMD $P_{2}$, Control vs. right TMD; $P_{3}$, Control vs. left TMD. ANOVA-Scheffé test.

\begin{tabular}{|c|c|c|c|c|c|c|}
\hline Control group & & Right TMD & Left TMD & $P 1$ & $P 2$ & $P 3$ \\
\hline Right temporalis & $286.39 \pm 26.09$ & $155.40 \pm 87.20$ & $228.31 \pm 30.49$ & 0.043 & 0.000 & 0.154 \\
\hline Right masseter & $268.70 \pm 34.45$ & $113.18 \pm 23.39$ & $186.90 \pm 26.28$ & 0.041 & 0.000 & 0.065 \\
\hline Left masseter & $297.88 \pm 33.35$ & $133.61 \pm 22.11$ & $170.66 \pm 27.44$ & 0.298 & 0.000 & 0.005 \\
\hline Left temporalis & $265.08 \pm 22.62$ & $185.92 \pm 25.95$ & $213.34 \pm 32.89$ & 0.516 & 0.026 & 0.201 \\
\hline
\end{tabular}

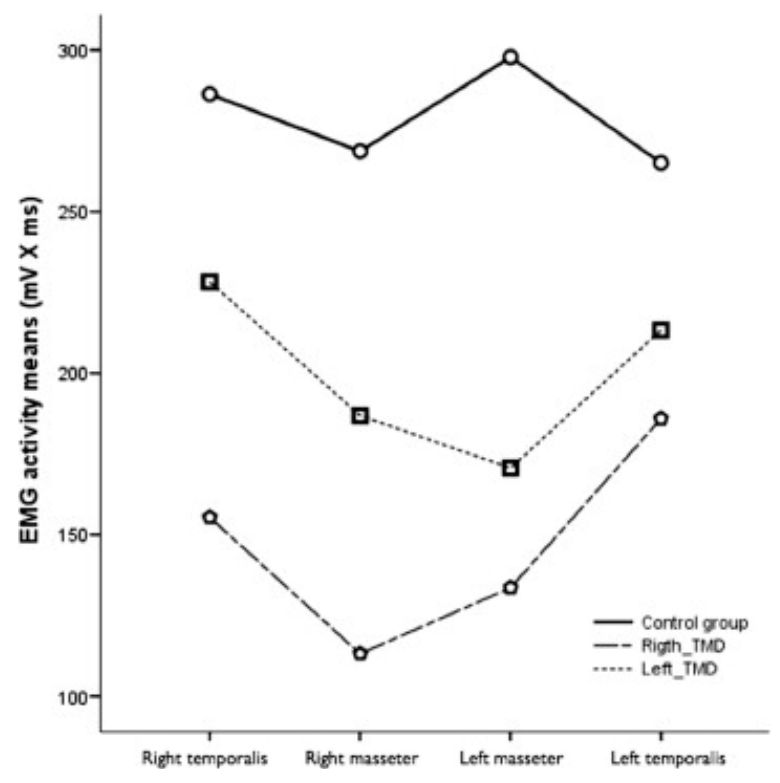

Fig. 3. EMG-activity of the four jaw muscles in the control and unilateral TMD subgroups.

3.2.3. EMG activity of right vs. left unilateral TMD patient's subgroups (Table 1 and Fig. 3).

Right temporalis and right masseters showed lower activity in the right- than in the leftTMD: right temporalis $155.40 \pm 87.20$ vs. $228.31 \pm 30.49, \quad P=0.043$; right masseter $113.18 \pm 23.39$ vs. $186.90 \pm 26.28, P=0.041$.

Patients with right-sided TMD demonstrated preferential use of their left-sided muscles (SAI $-5.35 \pm 4.02)$ whereas patients with left-sided TMD demonstrated preferential use of their rightsided muscles (SAI $6.95 \pm 2.82)$, $(P=0.016$, $t$-Student test).

\section{Discussion}

This is the first study to highlight the clinical significance of clench-EMG evaluations, i.e., the possibility of discriminating pain and non-pain sides with a clench-EMG in unilateral TMD patients with chronic pain, and it is of interest to researchers and clinicians who seek to obtain valuable information inherent in the EMG, while respecting the interpretation.

\subsection{Group selection}

It is reasonable to believe that the homogeneity of the sample enhances the internal validity of the study, thus some dependent variables (i.e., age, gender, hemispheric-dominance, and fulldentate normo-occlusion) have been excluded to homogenize both groups under study, which drastically reduced the sample size. The main negative consequence of such a homogeneous study design was that male patients were not evaluated and, due to gender differences in the central processing of the nociceptive input (Sarlani and Greenspan, 2005), these results should not be extrapolated to males a priori. 
The decision to limit the age range from 18 to 22 years was taken due to the age of the dentistry students, which also coincides with the peak of high prevalence of TMD (LeResche, 1997).

All subjects are Caucasian, and their height and weight are into the normality.

\subsection{EMG activity}

Due to the noise inherent with surface EMG, a special effort was made to obtain clean and reproducible standard records (Merletti, 1999). Approximately 20\% of the electrodes required careful relocation after new degreased, dry, jelly, and electrode fixation.

\subsubsection{Reproducibility}

The reproducibility was assessed in pain-free subjects to avoid possible interferences due to the patient's therapy. The results obtained regarding reproducibility were similar to those reported by Castroflorio et al. (2006).

\subsubsection{EMG activity in pain-free and unilateral TMD-pain patients}

The existence of a pathophysiologic link between clench-EMG activity and chronic TMD is still being debated. Several studies have shown that in clenching tasks, greater muscle activity involves greater bite-force generated by the elevator muscles and greater TMJ and/or tooth row loads (Hidaka et al., 1999, Van Eijden et al., 1993, Erhardson et al., 1993, Wood, 1987 and Van Spronsen et al., 1992). In agreement with other studies (Helkimo et al., 1975 and Fogle and Glaros, 1995), our findings showed low overall muscle activity in unilateral TMD patients ( $P=0.027$, Table 1$)$, which would suggest that a lower bite-force is to be expected.

Though Molin (1972) reported no differences in bite-force between affected and non-affected sides, our findings revealed differences between muscle activity on either side, i.e., activity was lower on the affected side, which would suggest less loading on the affected side. The discrepancy may be due to the fact that Molin registered biting forces exerted with the mandible in the habitual closing path and the teeth about $4 \mathrm{~mm}$ below the intercuspal position, whereas in our study EMG was registered with clenching in the maximal intercuspal position.

In spite of alterations in the neuromuscular system, no significant differences in clenching between patients with TMD and healthy controls have been reported (Nielsen et al., 1990). These observations are in disagreement with our findings, which can probably be explained in terms of differences in methodology; bilateral and TMD pain patients triggered by palpation were included and were not homogenized for age, gender, laterality, or occlusion.

Apparently, the right side TMD patients performed worse than the left side TMD ones. Although our work can not provide a direct explanation for this particular fact, it is tempting to speculate that the hemispheric dominance influence may play some role here (i.e., asymmetry of anatomic efferences from the central motor areas and tendency to develop one preferred chewing side). It deserves future research.

\subsection{Biomechanical considerations}

The question that remains to be answered is precisely how much of the generated loads are directed to the teeth and to the TMJ. Each subject may exert slightly different bite-forces and consequently tooth and/or TMJ loads in different clenching tasks, which may explain the variations in the intra-session EMG values, though they were not significantly different.

On the other hand, intrasession records avoid confounders such as different electrode location, differences between sides, and others. From the statistical point of view it is more correct to use the intrasession mean values. However, from a clinical point of view it seems easier to use a single record. We performed comparisons using one of the three records and similar results were found. In addition, the comparisons using non-parametric tests showed very similar significance. Thus, probably we can assume that it is enough to perform just one adequate, not-noised record by subject and session, which may be very important regarding the time and costs needed for EMG studies, particularly for large samples.

Clenching is a complex task and the biomechanism is as yet not fully understood. In all likelihood, there is an ideal situation in which clenching in the maximal intercuspal position (MIP) is balanced or coincident with centric occlusion (Academy of Prosthodontics, 2005), and 
it is plausible to believe that the loads are transmitted on the tooth-row. From a clinical point of view, this situation has only been observed in one non-pain subject. The direction of muscle forces is as important as force intensity and the location of muscle insertion points. A primary objective is to minimize TMJ loads by distributing them on the pairs of dental molars that are designed to receive the force in order to protect the TMJs. The temporalis muscle contract positions and elevates the mandible, but cannot generate an increase in TMJ loads, in particular, transdisc loads. The masseter muscles, nevertheless, stronger and more effective than temporalis muscles as they are shorter and closer to the tooth row, can produce forces that increase TMJ loading (Koolstra et al., 1988a, Koolstra et al., 1988b and Van Spronsen et al., 1996) that probably occurs when the jaw condyle is anteriorly displaced due to deflective occlusal contacts (Academy of Prosthodontics, 2005). In all likelihood, this would explain why patients exhibited less masseter muscle activity during clenching, particularly in the pain side masseter, which is, in accordance with previous reports (Lund et al., 1991 and Nickel et al., 2003), an effective protective mechanism for damaged TMJs. The specific recruitment of the masseter muscle appears to be the result of descending central modulation subsequent to nociceptive stimuli of the affected TMJ and/or myofascial and/or periodontal nociceptors (Sessle et al., 2000a). In contrast, the non-pain side temporalis was the relatively most active jaw muscle in unilateral TMD patients, and tended to reduce the compression of the affected TMJ as well as generating a tendency to rotate the mandible, producing a fulcrum in the healthy TMJ so that the contralateral jaw condyle (of the pain side) can advance, and avoid the compression of the bilaminar zone.

In short, muscle forces are directed to minimize joint loads and muscle efforts, indicating that it is a normal protective adjustment. This study showed the capacity of the masticatory apparatus to modulate muscle recruitment, thus reducing TMJ and/or tooth row loads on the pain-side by generating less activity on the ipsilateral masseter.

This unilateral reduction in temporalis and masseter activity is consistent with a conscious or subconscious effort to reduce joint loading on the pain side. This is a specific protective functional adaptation of the neuromuscular system due to nociceptive input.

\subsection{Methodological considerations and limitations}

According to the other authors (Glaros et al., 1997 and Gonzalez et al., 2008) one of the limitations of EMG diagnosis highlighted in this study is the inability to clearly distinguish pain from non-pain subjects.

Another limitation is the relatively small sample size. This is mainly due to the limited age range and the characteristics of the subject's occlusion (inclusion criteria). In our experience, EMG activity varied with age and occlusal condition (del Palomar et al., 2008); these variables should be taken in account in order to enhance the internal validity of the study.

A further limitation is the absence of an EMG examination of the internal muscles or those difficult to access, i.e., the pterygoid muscles, that undoubtedly can modify the vector force resulting from the complex functioning of masticatory muscles.

This study does not permit to elucidate if the reduction of EMG-activity was consequence of the efferent modulation directed to minimize TMJ-loading, or the functional impotence for the muscular ischemia, or both.

\subsection{Conclusion}

We conclude that EMG activity in unilateral TMD-pain patients was lower on the pain side than on the pain free side. The asymmetry index (SAI) may be a useful measure in discriminating patients with right vs. left-sided TMD.

Acknowledgements. The authors would like to thank the reviewer's recommendations and guidance that have contributed greatly to improve this article. We would also like to thank Dr. Thuan Dao for her valuable comments on a preliminary draft of this article.

This study was supported by Grant 9/7/1996/DOG/22/7/1996. Dirección Xeral de Universidades e Investigación of the Xunta de Galicia. 


\section{References}

Brooks et al., 1997. S.H. Brooks, J.W. Brand, S.J. Gibbs, L. Hollender, A.G. Lurie, K.A. Omnell, et al. Imaging of the temporomandibular joint. A position of the American Academy of Oral and Maxillofacial Radiology. Oral Surg Oral Med Oral Pathol Oral Radiol Endod, 83 (1997), pp. 609-618

Carlsson, 1983. A.M. Carlsson. Assessment of chronic pain I aspects of the reliability and validity of the visual analogue scale. Pain, 16 (1983), pp. 87-101

Carlsson and Ingervall, 1988. G.E. Carlsson, B. Ingervall. The dentition: occlusal variations and problems. N.D. Mohl, G.A. Zarb, G.E. Carlsson, J.D. Rugh (Eds.), A textbook of occlusion, Quintessence, Chicago (1988), pp. 209-226

Castroflorio et al., 2006. T. Castroflorio, K. Icardi, B. Becchino, E. Merlo, C. Debernardi, P. Bracco, et al. J Electromyogr Kinesiol, 16 (5) (2006), pp. 498-505 Epub 2005 Nov 15

Cram, 2004. J.R. Cram. Biofeedback applications. R. Merletti, Ph.A. Parker (Eds.), Electromyography, vol. 17Jonh Wiley and Sons, Inc., Hoboken, NJ (2004), pp. 435-451

del Palomar et al., 2008. A.P. del Palomar, U. Santana-Penín, M.J. Mora-Bermúdez, M. Doblaré. Clenching TMJs-loads increases in partial edentates: a 3D finite element study. Ann Biomed Eng, 36 (6) (2008), pp. 1014-1023 Epub 2008 Apr 4. PMID: 18389372, PubMed - in process

Dworkin and LeResche, 1992. S.F. Dworkin, L. LeResche. Research diagnostic criteria for temporomandibular disorders: Review, criteria, examinations and specifications, critique. J Craniomand Disord Facial Oral Pain, 6 (4) (1992), pp. 301-355

Erhardson et al., 1993. S. Erhardson, A. Sheikholeslam, C.M. Forsberg, P. Lockowandt. Vertical forces developed by the jaw elevator muscles during unilateral maximal clenching and their distribution on teeth and condyles. Swed Dent J, 17 (1-2) (1993), pp. 23-34

Farina et al., 2004. D. Farina, L. Arendt-Nielsen, R. Merletti, T. Graven-Nielsen. Effect of experimental muscle pain on motor unit firing rate and conduction velocity. J Neurophysiol, 91 (2004), pp. 1250-1259

Ferrario and Sforza, 1994. V.F. Ferrario, C. Sforza. Biomechanical model of the human mandible in unilateral clench: distribution of temporomandibular joint reaction forces between working and balancing sides. J Prosthet Dent, 72 (2) (1994), pp. 169-176

Fogle and Glaros, 1995. L.L. Fogle, A.G. Glaros. Contributions of facial morphology, age, and gender to EMG activity under biting and resting conditions: a canonical correlation analysis. J Dent Res, 74 (8) (1995), pp. 1496-1500

Glaros et al., 1997. A.G. Glaros, E.G. Glass, D. Brockman. Electromyographic data from TMD patients with myofascial pain and from matched control subjects: evidence for statistical, not clinical, significance. J Oorofac Pain, 11 (2) (1997), pp. 125-129

González et al., 2008. Y.M. González, C.S. Greene, N.D. Mohl. Technological devices in the diagnosis of temporomandibular disorders. Oral Maxillofac Surg Clin North Am, 20 (2) (2008), pp. 211-220 vi

Greene, 2006. C.h.S. Greene. Concepts of TMD etiology: effects on diagnosis and treatment. D.M. Laskin, C.h.S. Greene, W.L. Hylander (Eds.), Temporomandibular disorders, an evidence-based approach to diagnosis and treatment, Quintessence Publishing Co, Inc., Chandler Drivem IL, USA (2006), pp. 219-228 Helkimo et al., 1975. E. Helkimo, G.E. Carlsson, Y. Carmeli. Bite force in patients with functional disturbances of the masticatory system. J Oral Rehab, 2 (1975), pp. 397-406

Hidaka et al., 1999. O. Hidaka, M. Iwasaki, M. Saito, T. Morimoto. Influence of clenching intensity on bite force balance, occlusal contact area, and average bite pressure. J Dent Res, 78 (7) (1999), pp. 1336-1344

Johansson et al., 2003. A. Johansson, L. Unell, G.E. Carlsson, B. Söderfelt, A. Halling. Gender difference in symptoms related to temporomandibular disorders in a population of 50-year-old subjects. J Orofacial Pain, 17 (1) (2003), pp. 29-35

Kleinbaum et al., 1988. D.G. Kleinbaum, L.L. Kupper, K.E. Muller. Applied regression analysis and multivariable methods. PWS-KENT, Publishing Co. (1988) p. 17-2, 344-347

Koolstra et al., 1988a. J.H. Koolstra, T.M. Van Eijden, W.A. Weijs, M. Naeije. A three-dimensional mathematic model of the human masticatory system predicting maximum possible bite forces. J Biomech, 21 (7) (1988), pp. 563-576

Koolstra et al., 1988b. Koolstra JH, Van Eijden TMGJ, Weijs WA. Three-dimensional performance of the human masticatory system: the influence of the orientation and physological cross-section of the masticatory muscles. In: Biomechanics XI-A. Amsterdam: Free University Press; 1988b. p. 101-106.

LeResche, 1997. L. LeResche. Epidemiology of temporomandibular disorders: Implications for the investigation of etiologic factors. Crit Rev Oral Biol Med, 8 (3) (1997), pp. 291-305

Liljeström et al., 2005. M.-R. Liljeström, Y. Le Bell, P. Anttila, M. Aromaa, T. Jämsä, L. Metsähonkala, et al. Headache children with temporomandibular disorders have several types of pain and other symptoms. Cephalalgia, 25 (11) (2005), pp. 1054-1060

Lund et al., 1991. J.P. Lund, R. Donga, C.G. Widmer, C.S. Stohler. The pain-adaptation model: a discussion of the relationship between chronic musculoskeletal pain and motor activity. Can J Physiol Pharmacol, 69 (5) (1991), pp. 683-694

May and Garabadian, 2000. B.M. May, C. Garabadian. Reducing condylar compression in clenching patients. Crit Rev Biomed Eng, 28 (3-4) (2000), pp. 389-394

Merletti, 1999. Merletti R. Standards for Reporting EMG Data. International Society of Electrophysiology and Kinesiology. <https://www.isek-online.org/pdf/ISEK_EMG-Standards.pdf>, 1999. 
Milam et al., 1998. S.B. Milam, G. Zardeneta, J.P. Schmitz. Oxidative stress and degenerative temporomandibular joint disease: a proposed hypothesis. J Oral Maxillofac Surg, 56 (1998), pp. 214-223

Molin, 1972. C. Molin. Vertical isometric muscle forces of the mandible. A comparative study of subjects with and without manifest mandibular pain dysfunction síndrome. Acta Odont Scand, 30 (4) (1972), pp. 485-499

Møller and Bakke, 1988. E. Møller, M. Bakke. Occlusal harmony and disharmony: Frauds in clinical dentistry. Int Dent J, 38 (1) (1988), pp. 7-18

Merskey and Bogduk, 1994. H. Merskey, N. Bogduk. Classification of chronic pain: descriptions of chronic pain syndromes and definitions of pain terms. IASP Press, Seattle (1994)

Naeije et al., 1989. M. Naeije, R.S. McCarrol, W.A. Weijs. Electromographic activity of the human masticatory muscles during submaximal clenching in the intercuspal position. J Oral Rehab, 16 (1) (1989), pp. 63-70

Nielsen et al., 1990. I.L. Nielsen, C. McNeill, W. Danzig, S. Goldman, J. Levy, A.J. Miller. Adaptation of craniofacial muscles in subjects with craniomandibular disorders. Am J Orthod Dentofac Orthop, 97 (1) (1990), pp. 20-34

Nickel et al., 2003. J.C. Nickel, L.R. Iwasaki, R.D. Walker, K.R. McLachlan, W.D. McCall Jr. Human masticatory muscle forces during static biting. J Dent Res, 82 (3) (2003), pp. 212-217

Okeson, 1996. J.P. Okeson. Orofacial pain, guidelines for assessment, diagnosis, and management. The American academy of orofacial pain. Quintessence Publishing Co, Inc., Chicago IL (1996) pp 1-18, 11358

Oldfiel, 1971. R.C. Oldfiel. The assessment and analysis of handedness: the Edinburgh inventory. Nueropsychologia, 9 (1971), pp. 97-113

Pirttiniemi, 1998. P. Pirttiniemi. Normal and increased functional asymmetries in the craniofacial area. Acta Odontol Scand, 56 (6) (1998), pp. 342-345

Raadsheer et al., 1999. M.C. Raadsheer, T.M.G.J. Van Eijden, F.C. Van Ginkel, B. Prahl-Anmdersen. Contribution of jaw muscle size and craniofacial morphology to human bite force magnitude. J Dent Res, 78 (1) (1999), pp. 31-42

Racich, 2005. M.J. Racich. Orofacial pain and occlusion: Is there a link? An overview of current concepts and the clinical implications. J Prosthet Dent, 93 (2005), pp. 189-196

Sarlani and Greenspan, 2005. E. Sarlani, J.D. Greenspan. Why look in the brain for answers to temporomandibular disorder pain?. Cell Tissue Org, 180 (2005), pp. 69-75

Sessle, 2000a. B.J. Sessle. Acute and chronic craniofacial pain: brainstem mechanisms of nociceptive transmission and neuroplasticity, and their clinical correlates. Crit Rev Oral Biol Med, 11 (1) (2000), pp. 57-91

Sessle, 2000b. B.J. Sessle. Sex, gender, and pain. J Orofac Pain, 14 (3) (2000), p. 165

Smith et al., 1986. D.M. Smith, K.R. McLachlan, W.D. McCall Jr. A numerical model of temporomandibular join loading. J Dent Res, 65 (8) (1986), pp. 1046-1052

Suvinen and Kemppainen, 2007. T.I. Suvinen, P. Kemppainen. Review of clinical EMG studies related to muscle and occlusal factors in healthy and TMD subjects. J Oral Rehab, 34 (9) (2007), pp. 631-644

Svensson et al., 2004. P. Svensson, K. Wang, B.J. Sessle, L. Arendt-Nielsen. Associations between pain and neuromuscular activity in the human jaw and neck muscles. Pain, 109 (3) (2004), pp. 225-232

Tanaka et al., 2008. E. Tanaka, M.S. Detamore, L.G. Mercuri. Degenerative disorders of the temporomandibular joint: etiology, diagnosis, and treatment. J Dent Res, 87 (4) (2008), pp. 296-307

Academy of Prosthodontics, 2005. The Academy of Prosthodontics. The Glossary of prosthodontics terms. J Prosthet Dent, 94 (1) (2005), pp. 10-92

Van Eijden et al., 1993. T.M.G.J. Van Eijden, N.G. Blanksma, P. Brugman. Amplitude and timing of EMG activity in the human masseter muscle during selected motor tasks. J Dent Res, 72 (3) (1993), pp. 599-606

Van Spronsen et al., 1992. P.H. Van Spronsen, W.A. Weijs, J. Valk, B. Prahl-Andersen, F.C. Van Ginkel. A comparison of jaw muscle cross-sections of long-face and normal adults. J Dent Res, 71 (6) (1992), pp. 1279-1285

Van Spronsen et al., 1996. P.H. Van Spronsen, W.A. Weijs, F.C. Van Ginkel, B. Prahl-Andersen. Jaw muscle orientation and moment arms of long-face and normal adults. J Dent Res, 75 (6) (1996), pp. 13721380

Wood, 1987. W.W. Wood. A review of masticatory muscle function. J Prosthet Dent, 57 (2) (1987), pp. 222-232 